Article

\title{
An Algebraic Inequality with Applications to Certain Chen Inequalities
}

\author{
Ion Mihai ${ }^{1, *(\mathbb{D})}$ and Radu-Ioan Mihai ${ }^{2}$ \\ 1 Department of Mathematics, University of Bucharest, 010014 Bucharest, Romania \\ 2 Faculty of Mathematics and Computer Science, University of Bucharest, 010014 Bucharest, Romania; \\ radu.mihai4@s.unibuc.ro \\ * Correspondence: imihai@fmi.unibuc.ro
}

check for updates

Citation: Mihai, I.; Mihai, R.-I. An Algebraic Inequality with Applications to Certain Chen Inequalities. Axioms 2021, 10, 7. https://doi.org/10.3390/ axioms10010007

Received: 29 December 2020 Accepted: 7 January 2021 Published: 9 January 2021

Publisher's Note: MDPI stays neutral with regard to jurisdictional clai$\mathrm{ms}$ in published maps and institutional affiliations.

Copyright: (C) 2021 by the authors. Licensee MDPI, Basel, Switzerland. This article is an open access article distributed under the terms and conditions of the Creative Commons Attribution (CC BY) license (https:// creativecommons.org/licenses/by/ $4.0 /)$.
Abstract: We give a simple proof of the Chen inequality for the Chen invariant $\underbrace{\delta(2, \ldots, 2)}_{k \text { terms }}$ of submanifolds in Riemannian space forms.

Keywords: Riemannian space form; submanifold; Chen invariants; Chen inequalities

MSC: 53C40

\section{Introduction}

In [1,2], B.-Y. Chen introduced a string of Riemannian invariants, known as Chen invariants, which are different in nature from the classical Riemannian invariants. He established sharp relationships between these invariants and the squared mean curvature for submanifolds in Riemannian space forms, known as Chen inequalities (see [2]).

The proof uses an algebraic inequality, discovered by B.-Y. Chen in [1].

In the present paper, we obtain a different algebraic inequality that allows us to give simple proofs of certain Chen inequalities.

\section{Preliminaries}

The theory of Chen invariants and Chen inequalities was initiated by B.-Y. Chen [1,2]

Let $(M, g)$ be an $n$-dimensional $(n \geq 2)$ Riemannian manifold and $\nabla$ its Levi-Civita connection. One denotes by $R$ the Riemannian curvature tensor field on $M$. For any $p \in M$ and $\pi \subset T_{p} M$ a plane section, the sectional curvature $K(\pi)$ of $\pi$ is defined by $K(\pi)=R\left(e_{1}, e_{2}, e_{1}, e_{2}\right)$, where we use the convention $R\left(e_{1}, e_{2}, e_{1}, e_{2}\right)=g\left(R\left(e_{1}, e_{2}\right) e_{2}, e_{1}\right)$, with $\left\{e_{1}, e_{2}\right\}$ an orthonormal basis of $\pi$. Let $\left\{e_{1}, \ldots, e_{n}\right\}$ be an orthonormal basis of $T_{p} M$. The scalar curvature $\tau$ at $p$ is given by

$$
\tau(p)=\sum_{1 \leq i<j \leq n} K\left(e_{i} \wedge e_{j}\right)
$$

where $K\left(e_{i} \wedge e_{j}\right)$ is the sectional curvature of the plane section spanned by $e_{i}$ and $e_{j}$ (other authors consider $\left.\tau(p)=\sum_{1 \leq i \neq j \leq n} K\left(e_{i} \wedge e_{j}\right)\right)$.

The Chen first invariant $\bar{\delta}_{M}$ is defined by

$$
\delta_{M}(p)=\tau(p)-\inf \left\{K(\pi) \mid \pi \subset T_{p} M \text { plane section }\right\}
$$

The Chen invariant $\delta(2,2)$, given by

$$
\delta(2,2)(p)=\tau(p)-\inf \left\{K\left(\pi_{1}\right)+K\left(\pi_{2}\right) \mid \pi_{1}, \pi_{2} \subset T_{p} M \text { orthogonal plane sections }\right\},
$$

was studied in [3]. 
We shall consider the Chen invariant $\underbrace{\delta(2, \ldots, 2)}_{k \text { terms }}$, denoted by $\delta^{k}(2, \ldots, 2)$, which is given by

$$
\delta^{k}(2, \ldots, 2)(p)=\tau(p)-\inf \left\{K\left(\pi_{1}\right)+\cdots+K\left(\pi_{k}\right)\right\} ，
$$

where $\pi_{1}, \ldots, \pi_{k}$ are mutually orthogonal plane sections at $p$.

Obviously, $\delta^{1}(2)=\delta_{M}$.

In the next section, we shall prove an algebraic inequality and study its equality case. As an application we shall give a simple proof of the Chen inequality for the invariant $\delta^{k}(2, \ldots, 2)$.

\section{An Algebraic Inequality}

Proposition 1. Let $k, n \in \mathbb{N}^{*}, n \geq 2 k$, and $a_{1}, a_{2}, \ldots, a_{n} \in \mathbb{R}$. Then

$$
\sum_{1 \leq i<j \leq n} a_{i} a_{j}-\sum_{i=1}^{k} a_{2 i-1} a_{2 i} \leq \frac{n-k-1}{2(n-k)}\left(\sum_{i=1}^{n} a_{i}\right)^{2} .
$$

Moreover, the equality holds if and only if $a_{2 i-1}+a_{2 i}=a_{j}, 1 \leq i \leq k, 2 k+1 \leq j \leq n$.

Proof. We shall prove the above Proposition by mathematical induction.

Let

$$
P(n): \sum_{1 \leq i<j \leq n} a_{i} a_{j}-\sum_{i=1}^{k} a_{2 i-1} a_{2 i} \leq \frac{n-k-1}{2(n-k)}\left(\sum_{i=1}^{n} a_{i}\right)^{2},
$$

with equality holding if and only if $a_{2 i-1}+a_{2 i}=a_{j}, 1 \leq i \leq k, 2 k+1 \leq j \leq n$.

First we show that $P(2 k)$ is true. Indeed

$$
\begin{gathered}
\sum_{1 \leq i<j \leq 2 k} a_{i} a_{j}-\sum_{i=1}^{k} a_{2 i-1} a_{2 i} \leq \frac{k-1}{2 k}\left(\sum_{i=1}^{2 k} a_{i}\right)^{2} \Leftrightarrow \\
(k-1)\left(a_{1}^{2}+a_{2}^{2}+\cdots+a_{2 k}^{2}\right)-2 \sum_{1 \leq i<j \leq 2 k} a_{i} a_{j}+2 k\left(a_{1} a_{2}+a_{3} a_{4}+\cdots+a_{2 k-1} a_{2 k}\right) \geq 0 \Leftrightarrow \\
\left(a_{1}+a_{2}-a_{3}-a_{4}\right)^{2}+\cdots+\left(a_{1}+a_{2}-a_{2 k-1}-a_{2 k}\right)^{2}+\cdots+\left(a_{2 k-3}+a_{2 k-2}-a_{2 k-1}-a_{2 k}\right)^{2} \geq 0 .
\end{gathered}
$$

Clearly the equality holds if and only if $a_{1}+a_{2}=. .=a_{2 k-1}+a_{2 k}$.

Next, assume $P(n)$ and prove $P(n+1)$.

$$
P(n+1): \sum_{1 \leq i<j \leq n+1} a_{i} a_{j}-\sum_{i=1}^{k} a_{2 i-1} a_{2 i} \leq \frac{n-k}{2(n-k+1)}\left(\sum_{i=1}^{n+1} a_{i}\right)^{2},
$$

with equality holding if and only if $a_{2 i-1}+a_{2 i}=a_{j}, 1 \leq i \leq k, 2 k+1 \leq j \leq n+1$.

By using $P(n)$, one has

$$
\begin{gathered}
\sum_{1 \leq i<j \leq n+1} a_{i} a_{j}-\sum_{i=1}^{k} a_{2 i-1} a_{2 i}= \\
=\sum_{1 \leq i<j \leq n} a_{i} a_{j}+a_{n+1}\left(\sum_{i=1}^{n} a_{i}\right)-\sum_{i=1}^{k} a_{2 i-1} a_{2 i} \leq \\
\leq \frac{n-k-1}{2(n-k)}\left(\sum_{i=1}^{n} a_{i}\right)^{2}+a_{n+1}\left(\sum_{i=1}^{n} a_{i}\right) \leq
\end{gathered}
$$




$$
\leq \frac{n-k}{2(n-k+1)}\left(\sum_{i=1}^{n+1} a_{i}\right)^{2} .
$$

The last inequality is equivalent to

$$
\left[(n-k) a_{n+1}-\left(\sum_{i=1}^{n} a_{i}\right)\right]^{2} \geq 0 .
$$

The equality holds if and only if

$$
a_{2 i-1}+a_{2 i}=a_{j}, 1 \leq i \leq k, 2 k+1 \leq j \leq n+1 .
$$

\section{A Chen Inequality}

As an application of Proposition 1, we give a simple proof of the Chen inequality for the Chen invariant $\delta^{k}(2, \ldots, 2)$ of submanifolds in Riemannian space forms.

Let $\tilde{M}(c)$ be an $m$-dimensional Riemannian space form of constant sectional curvature c. The standard examples are the Euclidean space $\mathbb{E}^{m}$, the sphere $S^{m}$ and the hyperbolic space $H^{m}$.

Let $M$ be an $n$-dimensional submanifold of $\tilde{M}(c)$ and denote by $h$ the second fundamental form of $M$ in $\tilde{M}(c)$. Recall that the mean curvature vector $H(p)$ at $p \in M$ is given by

$$
H(p)=\frac{1}{n} \sum_{i=1}^{n} h\left(e_{i}, e_{i}\right)
$$

where $\left\{e_{1}, \ldots, e_{n}\right\}$ is an orthonormal basis of $T_{p} M$.

The submanifold $M$ is said to be minimal if $H(p)=0, \forall p \in M$.

The Gauss equation is (see [4])

$$
R(X, Y, Z, W)=c+g(h(X, Z), h(Y, W))-g(h(X, W), h(Y, Z)),
$$

for any vector fields $X, Y, Z, W$ tangent to $M$.

Theorem 1. Let $\tilde{M}(c)$ be an m-dimensional Riemannian space form of constant sectional curvature $c$ and $M$ an $n$-dimensional submanifold of $\tilde{M}(c)$. Then one has the following Chen inequality:

$$
\delta^{k}(2, \ldots, 2) \leq \frac{n^{2}(n-k-1)}{2(n-k)}\|H\|^{2}+\left[\frac{n(n-1)}{2}-k\right] c .
$$

Moreover, the equality holds at a point $p \in M$ if and only if there exist suitable orthonormal bases $\left\{e_{1}, \ldots, e_{n}\right\} \subset T_{p} M$ and $\left\{e_{n+1}, \ldots, e_{m}\right\} \subset T_{p}^{\perp} M$ such that the shape operators take the forms

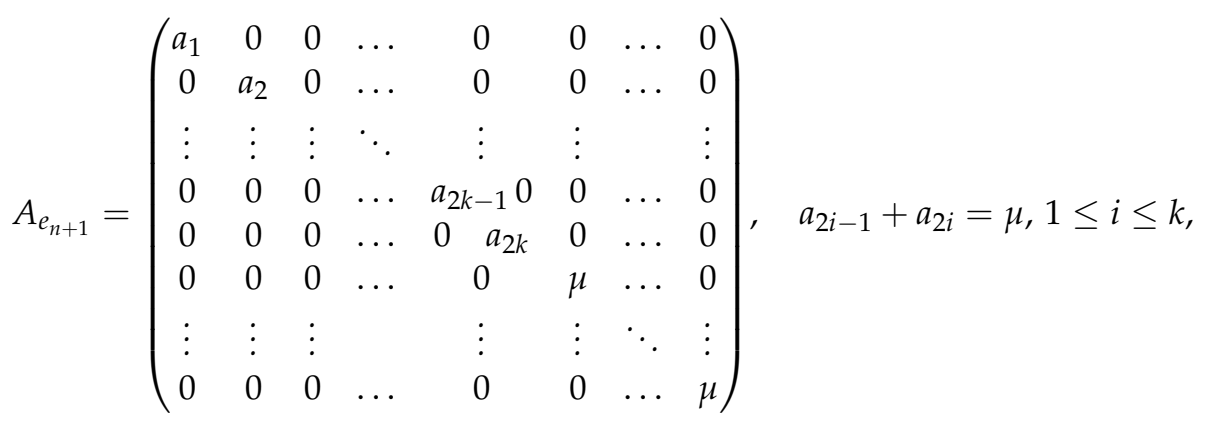




$$
A_{e_{r}}=\left(\begin{array}{ccccccc}
A_{1}^{r} & 0 & 0 & \ldots & 0 & 0 & 0 \\
0 & A_{2}^{r} & 0 & \ldots & 0 & 0 & 0 \\
\vdots & \vdots & \ddots & & \vdots & \vdots & \vdots \\
0 & 0 & 0 & A_{k}^{r} & 0 & 0 & 0 \\
0 & 0 & 0 & \ldots & 0 & 0 & 0 \\
\vdots & \vdots & \vdots & & \vdots & \ddots & \vdots \\
0 & 0 & 0 & \ldots & 0 & 0 & 0
\end{array}\right), \quad r=n+2, \ldots, m,
$$

where $A_{j}^{r}$ are symmetric $2 \times 2$ matrices with trace $A_{j}^{r}=0, \forall j=1, \ldots, k$.

Proof. Let $p \in M, \pi_{1}, \ldots, \pi_{k} \subset T_{p} M$ be mutually orthogonal plane sections and $\left\{e_{1}, e_{2}\right\} \subset$ $\pi_{1}, \ldots,\left\{e_{2 k-1}, e_{2 k}\right\} \subset \pi_{k}$ be orthonormal bases. We construct $\left\{e_{1}, \ldots, e_{2 k}, e_{2 k+1}, \ldots, e_{n}\right\} \subset$ $T_{p} M$ and $\left\{e_{n+1}, \ldots, e_{m}\right\} \subset T_{p}^{\perp} M$ orthonormal bases, respectively.

Denote by $h_{i j}^{r}=g\left(h\left(e_{i}, e_{j}\right), e_{r}\right), i, j=1, \ldots, n, r \in\{n+1, \ldots, m\}$, the components of the second fundamental form.

By the Gauss equation, we have

$$
\begin{aligned}
\tau & =\sum_{1 \leq i<j \leq n} K\left(e_{i} \wedge e_{j}\right)=\sum_{1 \leq i<j \leq n} R\left(e_{i}, e_{j}, e_{i}, e_{j}\right)= \\
& =\frac{n(n-1)}{2} c+\sum_{r=n+1}^{m} \sum_{1 \leq i<j \leq n}\left[h_{i i}^{r} h_{j j}^{r}-\left(h_{i j}^{r}\right)^{2}\right] .
\end{aligned}
$$

The Gauss equation also implies

$$
\begin{gathered}
K\left(\pi_{i}\right)=K\left(e_{2 i-1} \wedge e_{2 i}\right)=R\left(e_{2 i-1}, e_{2 i}, e_{2 i-1}, e_{2 i}\right)= \\
=c+\sum_{r=n+1}^{m}\left[h_{2 i-1,2 i-1}^{r} h_{2 i, 2 i}^{r}-\left(h_{2 i-1,2 i}^{r}\right)^{2}\right], \forall i=1, \ldots, k .
\end{gathered}
$$

Then we get

$$
\begin{gathered}
\tau-\sum_{i=1}^{k} K\left(\pi_{i}\right)= \\
=\left[\frac{n(n-1)}{2}-k\right] c+\sum_{r=n+1}^{m}\left[\sum_{1 \leq i<j \leq n} h_{i i}^{r} h_{j j}^{r}-\sum_{i=1}^{k} h_{2 i-1,2 i-1}^{r} h_{2 i, 2 i}^{r}\right]- \\
-\sum_{r=n+1}^{m} \sum_{\substack{1 \leq i<j \leq n,(i, j) \neq(1,2), \ldots,(2 k-1,2 k)}}\left(h_{i j}^{r}\right)^{2} .
\end{gathered}
$$

By using the algebraic inequality from the previous section, we obtain

$$
\begin{gathered}
\tau-\sum_{i=1}^{k} K\left(\pi_{i}\right) \leq \frac{n-k-1}{2(n-k)} \sum_{r=n+1}^{m}\left(\sum_{i=1}^{n} h_{i i}^{r}\right)^{2}+\left[\frac{n(n-1)}{2}-k\right] c= \\
=\frac{n^{2}(n-k-1)}{2(n-k)}\|H\|^{2}+\left[\frac{n(n-1)}{2}-k\right] c
\end{gathered}
$$

which implies the desired inequality.

If the equality case holds at a point $p \in M$, then we have equalities in all the inequalities in the proof, i.e.,

$$
\left\{\begin{array}{l}
h_{2 i-1,2 i-1}^{r}+h_{2 i, 2 i}^{r}=h_{j j}^{r}, 1 \leq i \leq k, 2 k+1 \leq j \leq n, \\
h_{i j}^{r}=0, \forall 1 \leq i<j \leq n,(i, j) \neq(1,2), \ldots,(2 k-1,2 k),
\end{array}\right.
$$


for any $r \in\{n+1, \ldots, m\}$.

We choose $e_{n+1}$ parallel to $H(p)$. Then the shape operators take the above forms.

Corollary 1. Let $\tilde{M}(c)$ be an m-dimensional Riemannian space form of constant sectional curvature $c$ and $M$ an $n$-dimensional submanifold of $\tilde{M}(c)$. If there exists a point $p \in M$ such that $\delta^{k}(2, \ldots, 2)(p)>\left[\frac{n(n-1)}{2}-k\right] c$, then $M$ is not minimal.

For $k=1$, one derives Chen's first inequality (see [1]).

Corollary 2. Let $\tilde{M}(c)$ be an m-dimensional Riemannian space form of constant sectional curvature $c$ and $M$ an n-dimensional submanifold of $\tilde{M}(c)$. Then one has

$$
\inf K \geq \tau-\frac{n-2}{2}\left[\frac{n^{2}}{n-1}\|H\|^{2}+(n+1) c\right] .
$$

Equality holds if and only if, with respect to suitable frame fields $\left\{e_{1}, \ldots, e_{n}, e_{n+1}, \ldots, e_{m}\right\}$, the shape operators take the following forms:

$$
\begin{gathered}
A_{e_{n+1}}=\left(\begin{array}{ccccc}
a & 0 & 0 & \ldots & 0 \\
0 & \mu-a & 0 & \ldots & 0 \\
0 & 0 & \mu & \ldots & 0 \\
\vdots & \vdots & \vdots & \ddots & \vdots \\
0 & 0 & 0 & \ldots & \mu
\end{array}\right), \\
A_{e_{r}}=\left(\begin{array}{ccccc}
h_{11}^{r} & h_{12}^{r} & 0 & \ldots & 0 \\
h_{12}^{r} & -h_{11}^{r} & 0 & \ldots & 0 \\
0 & 0 & 0 & \ldots & 0 \\
\vdots & \vdots & \vdots & \ddots & \vdots \\
0 & 0 & 0 & \ldots & 0
\end{array}\right), \quad r=n+2, \ldots, m .
\end{gathered}
$$

Example 1. The generalized Clifford torus.

$$
\text { Let } T=S^{k}\left(\frac{1}{\sqrt{2}}\right) \times S^{k}\left(\frac{1}{\sqrt{2}}\right) \subset S^{2 k+1} \subset \mathbb{E}^{2 k+2} \text {. }
$$
of $\mathbb{E}^{2 k+2}$.

It is known that $T$ is a minimal hypersurface of $S^{2 k+1}$, but a non-minimal submanifold

Obviously $\delta^{k}(2, \ldots, 2)=\tau=2 k(k-1)$.

Then $T \subset S^{2 k+1}$ does not satisfy the equality case of Theorem 1 .

If we consider $T \subset \mathbb{E}^{2 k+2}$, then it satisfies the equality case of Theorem 1 .

Remark 1. By using the inequality from Proposition 1, we can obtain Chen inequalities for the invariant $\delta^{k}(2, \ldots, 2)$ on submanifolds in other ambient spaces, for instance, complex space forms, Sasakian space forms, Hessian manifolds of constant Hessian curvature, etc.

Author Contributions: Conceptualization, I.M. and R.-I.M.; methodology, I.M.; software, R.-I.M.; validation, I.M. and R.-I.M.; formal analysis, I.M.; investigation, I.M. and R.-I.M.; resources, I.M.; data curation, I.M.; writing — original draft preparation, R.-I.M.; writing—review and editing, I.M. and R.-I.M.; visualization, I.M. and R.-I.M.; supervision, I.M.; project administration, I.M. and R.-I.M. All authors have read and agreed to the published version of the manuscript.

Funding: This research received no external funding.

Conflicts of Interest: The authors declare no conflict of interest. 


\section{References}

1. Chen, B.-Y. Some pinching and classification theorems for minimal submanifolds. Arch. Math. 1993, 60, 568-578. [CrossRef]

2. Chen, B.-Y. Some new obstructions to minimal and Lagrangian isometric immersions. Jpn. J. Math. 2000, 26, 105-127. [CrossRef]

3. Chen, B.-Y.; Prieto-Martin, A. Classification of Lagrangian submanifolds in complex space forms satisfying a basic inequality involving $\delta(2,2)$. Differ. Geom. Appl. 2012, 30, 107-123. [CrossRef]

4. Chen, B.-Y. Geometry of Submanifolds; M. Dekker: New York, NY, USA, 1973. 